H omer Yang MD CCFP FRCPC, Kristine Wilson-Yang PhD $M \mathrm{CIC}_{\text {, }}$ Karen Raymer MD FRCPC

\section{Why should we teach medical students?}

not a de facto reason for medical students to choose anesthesia as a career. H owever, in the same study, when the total number of students $(n=1480)$ who took the anesthesiology preceptorship were compared with the students who did not $(n=26,662)$, the proportions of students who entered anesthesia were $11.5 \%$ and $2.3 \%$ respectively; ${ }^{3}$ i.e., students who entered anesthesia were more likely to have taken an anesthesia preceptorship. The notion that exposure to anesthesia leads to increased recruitment would seem to be too complex an issue to gather clear evidence on a medical school by medical school basis. However, when viewed from a broader national perspective, with a larger sample size, the evidence exists to show that students who enter anesthesia are more likely to have taken an anesthesia preceptorship.

Another reason for anesthesiologists to teach medical students may be to influence student attitudes towards anesthesia. In one American study, the presence of certified registered nurse anesthetists was found to be a negative factor in medical students' career choice for anesthesia. ${ }^{4}$ In another study, a survey of ten medical schools was conducted. Of the four medical schools whose students performed best on the anesthesia knowledge quiz, two provided routine exposure to anesthesia while the other two instituted a one-on-one preceptor-student relationship. ${ }^{5} \mathrm{M}$ ore students in the schools with the routine anesthesia exposure had a negative attitude towards anesthesia. A nother study found that the attitudes towards anesthesia after the clerkship rotation was more positive. ${ }^{6}$ It would seem that student attitudes towards anesthesia after an anesthesia rotation are perhaps more dependent on local factors within a medical school.

In part, this is an issue of quantity vs quality. From the perspective of individual medical schools, there are no studies correlating the quality of exposure to the recruitment rate. From the perspective of role models, a recent study in Australia found that for those students who intended to enter anesthesia (18\%), 94\% identified a positive role model compared to $65 \%$ for 
who intended otherwise $(P=0.03) \cdot{ }^{7}$ In a study of clerks rotating through internal medicine, exposure to the "best" teachers did not influence residency choice while exposure to the "worst" teachers was associated with being less likely to choose a residency in internal medicine. ${ }^{8}$ In a subsequent paper, the authors found that " better medical students who work with the best internal medicine attending physicians and residents .... are more likely to choose an internal medicine residency." 9 To no one's surprise, the evidence supports the idea that the quality of exposure matters, especialIy if a specialty were to attract the best students. In that context, our study in 1994 found that only $4 \%$ of the postgraduate trainees in anesthesia at that time chose anesthesia due to a positive role model. ${ }^{2}$ The study, as in many similar studies, had limitations, but does leave one to speculate about the specialty's involvement in UME at that time. To date, there have been no repeat studies on postgraduate trainees and their reasons for choosing or rejecting anesthesia as a career in Canada.

It is important to realize that factors such as faculty role models and curriculum organization are considered to be medical school characteristics. Those characteristics are one of three groups of factors influencing a student's career choice. The others are a medical student's personal characteristics, i.e., in search of a controllable lifestyle, ${ }^{10-12}$ marital status, ${ }^{13}$ rural background, ${ }^{14}$ and geographic location; ${ }^{15}$ and a specialty's characteristics such as intellectual content, ${ }^{16,17}$ income potential, ${ }^{18,19}$ and prestige. ${ }^{17,19}$ It is generally agreed that personal or specialty factors are not modifiable.

In order to provide more information on specialty characteristics, career counselling workshops are offered by many medical schools in North America. The Pathways Workshop sponsored by Glaxo Wellcome, for example, has been offered since the early 1990s. The Pathways workshop provides a specialty profile booklet which allows medical students to review and compare what practitioners in the various specialties say about their own specialties. From the most recent edition, the relative value practitioners place on intellectual content (i.e., specific problemsolving vsthe examination of larger theoretical issues), importance of income (i.e., income levels being a consideration in satisfaction of practice), and respect / approval by others (i.e., the expectation of, or need for, respect and approval from others) are shown in the Table. The rating by anesthesiologists on intellectual content was the lowest among the specialties in the Table. Although not scientific, the values shown are those reported by the practitioners interviewed in
T A B L E R elative importance as reported by practitioners in each specialty ${ }^{2}$

\begin{tabular}{llll}
\hline $\begin{array}{l}\text { (1 being least important, } 10 \text { most important) } \\
\text { Intellectual } \\
\text { Content }\end{array}$ & Income & $\begin{array}{l}\text { R espect \& } \\
\text { A pproval }\end{array}$ \\
\hline Family M edicine & 4.2 & 4.5 & 6.8 \\
Anesthesia & 3.2 & 6.9 & 5.7 \\
Cardiology & 4.1 & 7.0 & 7.7 \\
D ermatology & 3.3 & 5.8 & 5.9 \\
H ematology & 4.9 & 5.6 & 7.6 \\
Internal M edicine & 4.2 & 4.8 & 7.4 \\
O b / Gyne & 3.4 & 6.7 & 7.4 \\
Psychiatry & 6.2 & 4.5 & 5.4 \\
Radiology & 3.4 & 6.8 & 7.3 \\
Surgery & 4.1 & 6.4 & 7.5 \\
\hline
\end{tabular}

each specialty, including anesthesia. More importantly, those are the values taken away by many students in $\mathrm{N}$ orth America in their decision making process for their career choice. In the absence of direct contact with our specialty, medical students will form their impressions based on other sources of information, such as the G laxo Pathways workshop.

Among personal factors, the search for a controllable lifestyle was a significant factor influencing medical student career choices in the $1980 \mathrm{~s}^{10,11}$ In the 1970s, a specialty's characteristics were considered to be more important. ${ }^{10} \mathrm{C}$ urrently, there is an increase in the number of applicants for anesthesia in Canada. Although unproven, the general feeling is that the shortage of anesthesiologists and the almost assured availability of a consultant post after residency have been instrumental in the increase in applicant numbers. Whatever the factors considered important by medical students in making their career choices, it is important to note that those considerations are cyclical: they have changed and will change from decade to decade, if not from year to year. As a specialty, it would be unwise to formulate policies guiding the teaching of medical students based on the current applicant numbers.

An often cited barrier for anesthesiologists teaching medical students is the minimal or lack of remuneration for individuals who participate in UME. Not infrequently, the lack of remuneration is perceived to be a problem unique to one's own department. A recent paper reported the results of a cross-sectional survey of U S internal medicine teaching units. ${ }^{20}$ It is interesting and unfortunate to see that half of the teaching faculty were unfunded by the teaching unit. The authors felt that the real costs of medical education may be considerably underestimated, necessitating continued dependence on volunteer faculty. 
"Funding and professional recognition may be needed to continue...."

It is clear that the underfunding of medical education is not unique to anesthesia and the degree of underfunding is huge. It is an issue that must be addressed but may take years, if not decades to resolve. In the meantime, the specialty of anesthesia, along with the anesthesia department of each teaching centre, will need to find the solution or solutions, be it the procurement of more funding, redistribution of resources within the group to support teaching, or the elimination of UME teaching altogether. In light of the impact of good teachers and positive anesthesia rotations on medical students, especially the better students, the last option would have a tremendous negative impact on the future of our specialty.

When all is said and done, UME will require some soul searching within each and every one of us. Through teaching our specialty stands to gain: positive role models will help to attract the best students into the specialty. Unfortunately, evidence also suggests that most of the teaching is done on a voluntary basis, by people who receive little or no direct benefit to themselves, apart from the recognition and appreciation of their students. U nderfunding alone, however, does not explain the limited presence of anesthesia in UME- medicine as a whole has a long, proud history of voluntarism in teaching. The real question then becomes, "Do we believe that we possess an essential body of knowledge and skills that we can and should impart to future generations of physicians?" If so, do we believe this strongly enough to surmount the obstacles that stand before us?

\section{Pourquoi enseigner aux étudiants en médecine?}

La revue du présent numéro a été réalisée pour l'occasion par Richard Brull et John Bradley ${ }^{1}$ et s'intitule "The role of anesthesiologists in $\mathrm{C}$ anadian undergraduate medical education" (Le rôle des anesthésiologistes dans la formation des étudiants en médecine au $\mathrm{C}$ anada). O n y examine la question fort à propos de la participation des anesthésiologistes à la formation des étudiants en médecine (FEM). Les résultats montrent que treize écoles de médecine sur seize exigent une rotation en anesthésie pendant les stages, mais que peu d'anesthésiologistes participent à la formation d'avant-stage. On peut penser que les écoles de médecine méconnaissent les avantages pédago giques de la participation des anesthésiologistes à la FEM ou que les anesthésiologistes sont eux-mêmes réticents à assumer les responsabilités de I'enseignement d'avant-stage. Cette dernière affirmation peut laisser croire que les anesthésiologistes considèrent que l'enseignement aux étudiants en médecine n'en vaut pas la peine. Dans le présent éditorial, nous chercherons à savoir pourquoi les anesthésiologistes devraient enseigner aux étudiants en médecine.

La participation des anesthésiologistes au pré-stage peut contribuer à rassurer les étudiants en médecine sur le choix éclairé d'une carrière possible en anesthésie. Selon l'actuel Canadian Residents $M$ atching Service ( $C$ aR M S), les étudiants doivent faire part de leur choix de spécialité en octobre de l'année de leur stage quoique le choix final puisse être modifié, non sans difficulté, jusqu'en janvier. Comme le stage se poursuit habituellement jusqu'en avril ou mai, une grande partie des étudiants doivent faire leur choix définitif avant la rotation en anesthésie.

$L$ 'une des raisons, pour les anesthésiologistes, d'enseigner aux étudiants en médecine serait d'augmenter le recrutement, ce qu'il convient alors de vérifier. Ainsi, au cours de la précédente enquête nationale, en 1994, la durée d'un stage en anesthésie dans une école de médecine n'était pas en corrélation avec le nombre d'étudiants de cette école à s'inscrire en anesthésie. ${ }^{2} D$ ans une enquête menée dans 76 écoles de médecine américaines qui offraient un stage en anesthésiologie, le pourcentage, par école, de médecins inscrits dans la spécialité après y avoir fait un stage, allait de 0 à $80 \%^{3}$ O n pourrait donc penser que la simple exposition à l'anesthésie n'est pas une raison de facto d'y faire carrière. Cependant, dans la même étude, si on compare le nombre total d'étudiants ( $n=1480$ ) qui ont choisi le stage à celui des étudiants qui ne l'ont pas choisi ( $n=26$ 662), la proportion des étudiants qui se sont inscrits en anesthésie a été de $11,5 \%$ et de 2,3\% respectivement; 3 c'est donc dire que les étudiants qui choisissent l'anesthésie sont plus susceptibles d'y avoir fait un stage. L'analyse, par école, de la prémisse voulant que l'exposition à l'anesthésie conduise à un meilleur recrutement semblait trop complexe pour fournir quelque indication que ce soit sur une école de médecine. Par contre, dans une perspective nationale, un échantillon plus important illustre, à l'évidence, que les étudiants qui s'inscrivent en anesthésie sont plus susceptibles d'avoir participé à un stage en anesthésie.

$U$ ne autre raison d'enseigner aux étudiants en médecine, serait d'influencer les attitudes face à I'anesthésie. Dans une étude américaine, la présence 
TABLE Importance relative assignée par les praticiens de chaque spécialité 21

\begin{tabular}{llll}
\hline \multicolumn{4}{c}{ (1 étant le moinsimportant et 10, le plusimportant) } \\
& $\begin{array}{l}\text { Contenu } \\
\text { intellectud }\end{array}$ & R evenu & $\begin{array}{l}\text { R espect \& } \\
\text { R econnaissance }\end{array}$ \\
\hline M édecine familiale & 4,2 & 4,5 & 6,8 \\
Anesthésie & 3,2 & 6,9 & 5,7 \\
Cardiologie & 4,1 & 7,0 & 7,7 \\
Dermatologie & 3,3 & 5,8 & 5,9 \\
H ématologie & 4,9 & 5,6 & 7,6 \\
M édecine interne & 4,2 & 4,8 & 7,4 \\
Gyn / Ob & 3,4 & 6,7 & 7,4 \\
Psychiatrie & 6,2 & 4,5 & 5,4 \\
Radiologie & 3,4 & 6,8 & 7,3 \\
Chirurgie & 4,1 & 6,4 & 7,5 \\
\hline
\end{tabular}

d'infirmières anesthésistes diplômées a été vue comme un facteur négatif dans le choix de cette spécialité par les étudiants en médecine. ${ }^{4} \mathrm{D}$ ans une autre étude, une enquête a été menée dans dix écoles de médecine. $D$ es quatre écoles de médecine où les étudiants ont obtenu les meilleurs résultats à un jeu-questionnaire sur I'anesthésie, deux offraient une formation de routine en anesthésie et les deux autres avaient instauré une relation exclusive tuteur-étudiant. ${ }^{5} \mathrm{U}$ n plus grand nombre d'étudiants ayant fait le stage de routine en anesthésie avaient une attitude négative face à l'anesthésie. U ne autre étude a fait ressortir que les attitudes envers l'anesthésie étaient plus positives après y avoir fait un stage de rotation. ${ }^{6}$ II semble que, dans ces circonstances, les attitudes des étudiants peuvent être plus dépendantes de facteurs particuliers à une école.

$C^{\prime}$ 'est donc, en partie, une question de quantité vs qualité. Du point de vue des écoles de médecine prises séparément, il n'existe pas d'études qui établissent une corrélation entre la qualité du stage et le taux de recrutement. En regard de la notion de modèle, une récente étude faite en Australie a montré que parmi les étudiants qui pensent s'inscrire en anesthésie (18\%), $94 \%$ ont déclaré que le stage représentait un modèle positif de la profession, en comparaison avec $65 \%$ des étudiants qui pensent s'inscrire ailleurs $(P=0,03)^{7}$. $D$ ans une étude auprès de stagiaires en rotation en médecine interne, le contact avec les "meilleurs" professeurs n'a pas influencé le choix de la résidence tandis que le contact avec les "pires" professeurs a été associé à une moins forte incitation à choisir une résidence en médecine interne. ${ }^{8} \mathrm{D}$ ans un article subséquent, les auteurs ont trouvé que "les meilleurs étudiants en médecine qui travaillent avec les meilleurs internistes et résidents de la spécialité... sont plus susceptibles de choisir une résidence en médecine interne." 9 É videmment, les faits confirment l'idée que la qualité du contact est importante, surtout si on veut attirer les meilleurs étudiants dans la spécialité. $D$ ans ce contexte, notre étude de 1994 a montré que seulement $4 \%$ des stagiaires en anesthésie avaient choisi cette spécialité à cause d'un modèle positif. ${ }^{2}$ L'étude, comme beaucoup d'autres études similaires, avait ses limites, mais amenait certainement à réfléchir sur la participation de la spécialité à la FEM de l'époque. J usqu'à aujourd'hui, il n'y a pas eu d'autres études sur les résidents et sur les raisons qui les ont motivés à choisir ou à rejeter l'anesthésie pour faire carrière au Canada.

II est important de se rappeler que les modèles de professeurs et le programme d'enseignement sont considérés comme des caractéristiques d'une école de médecine. Ces caractéristiques représentent I'un des trois groupes de facteurs d'influence sur le choix de carrière d'un étudiant. Les autres étant les caractéristiques personnelles de l'étudiant, c'est-à-dire, la recherche d'une certaine qualité de vie, ${ }^{10-12}$ la situation de famille, ${ }^{13}$ l'origine rural $e^{14}$ et la situation géographique; ${ }^{15}$ enfin, les caractéristiques de la spécialité comme le contenu intellecuel, ${ }^{16,17}$ le revenu possible ${ }^{18,19}$ et le prestige. ${ }^{17,19} \mathrm{E} n$ général, on s'entend pour dire que les facteurs personnels et ceux qui sont reliés à la spécialité ne sont pas modifiables.

$\mathrm{D}$ ans le but de mieux décrire les caractéristiques de la spécialité, des ateliers d'orientation professionnelle sont offerts par de nombreuses écoles de médecine en A mérique du Nord. Ainsi, le Pathways Workshop parrainé par Glaxo Wellcome existe depuis le début des années 1990. L'atelier Pathways fournit une brochure qui présente la spécialité, les étudiants en médecine peuvent ainsi revoir et comparer ce que disent les praticiens de diverses spécialités au sujet de leur domaine de pratique. $D$ ans la dernière édition, la valeur relative accordée par praticiens au contenu intellectuel (vu comme la résolution de problèmes spécifique vs l'examen de questions théoriques plus vastes), à l'importance du revenu (l'échelle de revenu considérée comme un aspect satisfaisant de l'exercice de la profession) et au respect / reconnaissance des autres (donc, les attentes et les besoins par rapport au respect et à l'approbation des autres) est illustrée dans la Table. La cote du contenu intellectuel par les anesthésiologistes est la plus basse, parmi les spécialités mentionnées. Quoique non scientifique, les valeurs illustrées sont celles qui ont été mentionnées par les praticiens interviewés dans chaque spécialité, dont I'anesthésie. Q ui plus est, ces valeurs sont retenues par de nombreux étudiants d'Amérique du $\mathrm{N}$ ord lors $\mathrm{du}$ choix de carrière. Faute de contact direct avec notre 
spécialité, les étudiants devront fonder leurs impressions sur d'autres sources de renseignements, comme I'atelier de Glaxo Pathways.

Parmi les facteurs personnels, la recherche d'une qualité de vie exerçait une influence significative sur le choix de carrière de l'étudiant en médecine dans les années 1980.10,11 D ans les années 1970, les caractéristiques d'une spécialité étaient considérées comme plus importantes..$^{10}$ Actuellement, le nombre d'inscriptions en anesthésie augmente au Canada. Bien que ce ne soit pas prouvé, on croit généralement que la pénurie d'anesthésiologistes et la possibilité presque assurée d'un poste de consultant après la résidence ont joué un rôle décisif dans l'augmentation des inscriptions. Q uels que soient les facteurs retenus lors du choix de carrière des étudiants en médecine, ils demeurent cycliques, changeant d'une décennie à l'autre, sinon d'une année à l'autre. Par conséquent, on ne peut, comme spécialité, orienter l'enseignement des étudiants en médecine en se fondant sur le nombre actuel d'inscriptions.

L'obstacle souvent cité par les anesthésiologistes à la formation des étudiants en médecine est la rémunération minimale ou même l'absence de rémunération de ceux qui y participent. II n'est pas rare que cette situation soit perçu comme un problème unique à son propre département. Un article récent rapporte les résultats d'une enquête transversale menée dans les unités de formation américaines en médecine interne. ${ }^{20}$ II est intéressant, mais regrettable, de constater que la moitié des professeurs n'étaient pas rémunérés par l'unité de formation. Les auteurs croient que le coût réel de la formation médicale est sous-estimé et qu'il faut sans cesse compter sur un professorat bénévole. "L e financement et la reconnaissance professionnelle pourraient être nécessaires pour continuer..."

II est clair que le sous-financement de la formation médicale n'est pas unique à l'anesthésie et que son niveau est élevé. II faut résoudre ce problème, ce qui pourrait exiger des années, voire des décennies. Entretemps, la spécialité de l'anesthésie, avec le département d'anesthésie de chaque centre de formation, devra trouver la solution ou les solutions, que ce soit un financement accru, une redistribution des ressources à l'intérieur du groupe pour soutenir l'enseignement ou l'élimination radicale de la participation au programme de FEM. Selon l'impact reconnu de la présence de bons professeurs et de rotations positives en anesthésie sur les étudiants en médecine, surtout sur les meilleurs d'entre eux, la dernière option pourrait avoir un effet négatif très important sur l'avenir de notre spécialité.
Tout bien considéré, la FEM aura besoin d'un examen de conscience de chacun d'entre nous. Notre spécialité a des chances de profiter de l'enseignement, car les modèles positifs attirent les meilleurs étudiants dans la spécialité. $M$ alheureusement, les faits indiquent que la majeure partie de l'enseignement relève de la collaboration volontaire de gens qui reçoivent peu ou pas de bénéfices pour eux-mêmes, sauf la reconnaissance et l'appréciation de leurs étudiants. Le sous-financement seul ne peut toutefois expliquer la présence limitée de l'anesthésie dans la FEM - dans l'ensemble, la médecine s'enorgueillit d'une longue histoire de bénévolat en enseignement. La question réelle devient alors, "Croyons-nous que nous possédons un bagage essentiel de connaissances et de compétences que nous pouvons et que nous devrions transmettre aux générations futures de médecins?" Si c'est le cas, y croyons-nous suffisamment pour surmonter les obstacles qui se dressent devant nous?

\section{References}

1 Brull R, Bradley JW. The role of anesthesiologists in Canadian undergraduate medical education. Can J Anesth 2001; 48: 147-52.

$2 \mathrm{Y}$ ang $\mathrm{H}$, Wilson-Yang $\mathrm{K}, \mathrm{R}$ aymer $\mathrm{K}$. Recruitment in anaesthesia: results of two national surveys. Can J Anaesth 1994; 41: 621-7.

3 Bruhn JG, Epstein BS, Burnap TK. Specialty choices of medical graduates taking anesthesiology preceptorships: a follow-up study. Anesthesiology 1974; 41: 270-4.

$4 \mathrm{C}$ handra $\mathrm{P}, \mathrm{H}$ ughes $\mathrm{M}$. Factors affecting the choice of anesthesiology by medical students for specialty training. J Med Educ 1984; 59: 323-30.

5 Bruhn JG, Epsten BS, Burnap TK. Senior medical students' knowledge of and attitudes toward anesthesiology in ten medical schools. Anesthesiology 1973; 39: 94-103.

6 Samra SK, Davis W, Pandit SK, Cohen PJ. The effect of a clinical clerkship on attitudes of medical students toward anesthesiology. J M ed Educ 1983; 58: 641-7.

7 Watts R W, Marley J, W orley P. U ndergraduate education in anaesthesia: the influence of role models on skills learnt and career choice. Anaesth Intensive $\mathrm{C}$ are 1998; 26: 201-3.

8 Griffith CH III, Wilson JF, H aist SA, R amsbottomLucier M. Relationships of how well attending physicians teach to their students' performances and residency choices. Acad M ed 1997; 72: S118-20.

9 Griffith CH III, Georgesen JC, Wilson JF. Specialty choices of students who actually have choices: the influence of excellent clinical teachers. Acad M ed 2000; 75 : 278-82.

10 Schwartz R W, Jarecky R K, Strodel WE, H aley JV, Y oung 
B, Griffen WO Jr. Controllable lifestyle: a new factor in career choice by medical students. Acad M ed 1989; 64: 606-9.

11 Schwartz RW, Simpson WG, Strodel WE, Jarecky RK, Griffen WO J r, Y oung AB. Career change: in quest of a controllable lifestyle. J Surg Res 1989; 47: 189-92.

12 SimmondsAC IV, RobbinsJM, Brinker MR, RiceJC, $K$ erstein MD. Factors important to students in selecting a residency program. Acad M ed 1990; 65: 640-3.

$13 \mathrm{~N}$ ewton DA, Grayson MS, Whitley TW. What predicts medical student career choice? J Gen Intern M ed 1998; 13: 200-3.

14 Peach H G, Bath NE. Comparison of rural and nonrural students undertaking a voluntary rural placement in the early years of a medical course. M ed Educ 2000; 34: 231-3.

15 R ose SH , Faust R J, Milde LN , Wedel DJ. Factors affecting choice of residency in anesthesiology. Anesth Analg 1990; 70: S330.

16 Burack JH, I rby D M , Carline J D, A mbrozy D M, Ellsbury KE, Stritter FT. A study of medical students' specialty-choice pathways: trying on possible selves. Acad M ed 1997; 72: 534-41.

17 Schafer S, Shore W, French L, Tovar J, H ughes S, H earst $\mathrm{N}$. Rejecting family practice: why medical students switch to other specialties. Fam M ed 2000; 32: 320-5.

$18 \mathrm{~K}$ asster WJ, Wartman SA, Silliman R A. Why medical students choose primary care careers. A cad M ed 1991; 66: 41-3.

19 R ogers LQ, Fincher R M, Lewis LA. Factors influencing medical students to choose primary care or non- primary care specialties. Acad M ed 1990; 65: S47-8.

$20 \mathrm{~N}$ elson H D, Cooney TG, Kroenke K, Friedman R H. Contributions of general internal medicine teaching units: a national survey. J Gen Intern M ed 2000; 15: 277-83.

21 A nonymous Specialty Profiles. Glaxo Pathway Evaluation Program. M ississauga: Glaxo Canada Inc., 1999. 